\title{
The significance of nanoparticles in particle- induced pulmonary fibrosis
}

\author{
James D Byrne*, John A Baugh
}

\begin{abstract}
Exposure to airborne nanoparticles contributes to many chronic pulmonary diseases. Nanoparticles, classified as anthropogenic and natural particles, and fibers of diameters less than 100 $\mathrm{nm}$, have unrestricted access to most areas of the lung due to their size. Size relates to the deposition efficiency of the particle, with particles in the nano-range having the highest efficiencies. The deposition of nanoparticles in the lung can lead to chronic inflammation, epithelial injury, and further to pulmonary fibrosis. Cases of particle-induced pulmonary fibrosis, namely pneumoconiosis, are mostly occupationally influenced, and continue to be documented around the world. The tremendous growth of nanotechnology, however, has spurred fears of increased rates of pulmonary diseases, especially fibrosis. The severity of toxicological consequences warrants further examination of the effects of nanoparticles in humans, possible treatments and increased regulatory measures.
\end{abstract}

KEYWORDS: Nanoparticles, fibrosis, silicosis, asbestosis, pneumoconiosis, nanotubes

\section{INTRODUCTION}

The majority of existing research on particulate causes of pulmonary fibrosis focuses generally on the micron-scale. This review focuses more specifically on nano-scale particles (particles and fibers with diameters less than $0.1 \mu \mathrm{m}$ ), or nanoparticles. In vitro and in vivo studies support that nanoparticles are significant contributors to pulmonary fibrosis, a debilitating condition often leading to death (1). These particles are present in occupational and environmental settings at concentrations dependent upon location, season, and time of day.

Clinical cases of particle-induced pulmonary fibrosis are nearly all occupational in origin. An epidemiological study in Mongolia from 1967-2004 showed that $67.8 \%$ of cases involving occupational diseases were diagnosed as dust-induced chronic bronchitis and pneumoconiosis (2). Developing countries continue to be plagued with such cases of pulmonary fibrosis and other chronic pulmonary diseases caused by particles. Recently, China has experienced 10,000 to 15,000 new cases of pneumoconiosis per year, which has increased yearly since 1949. Between 1949 and 2001, China has

*To whom correspondence should be addressed: James Byrne

2600 Rio Grande St., Austin, TX, USA, 78705

Email: james.byrne@mail.utexas.edu recorded 569,129 confirmed cases of pneumoconiosis (3). Canada and the U.S. have seen decreasing trends in both diagnosis and mortality rates involving occupational cases of particle-induced pulmonary fibrosis (4). In the U.S., an epidemiological study from 1968-2000 showed that 124,849 deaths were attributed to pneumoconiosis (5). Developed nations experience decreasing trends as a result of improved hygiene conditions in mines, superior dust control, and better use of respiratory protective measures (4). The rapid increase in nanotechnology worldwide has the potential to dramatically increase the exposure of humans to uncharacterized particles and cause an increase in pulmonary disease in many developed areas. For example, engineered nanoparticles, such as carbon nanotubes, have been shown to cause fibrosis in rats (6). The severity of nanoparticle effects warrants further elucidation and increased regulatory measures to protect against human exposure.

\section{EXPOSURE TO AIRBORNE NANOPARTICLES}

Exposure to airborne nanoparticles is an unavoidable consequence of the technological advances of combustion engines and engineering and the natural occurrence of dusts, forest fires, and volcanic activity. As the most toxic component of airborne particulate matter, nanoparticles have uncontrolled access to the cells of the airway and even intracellular components 
because of their size (7-9). Central to pulmonary toxicity is nanoparticle deposition in the alveolar spaces of the lung. Nanoparticles have high deposition efficiencies in the lungs of healthy individuals, and even higher efficiencies in individuals with asthma or chronic obstructive pulmonary disease $(10,11)$.

When inhaled, nanoparticles deposit dispersedly upon the alveolar surface, which likely leads to a scattered chemoattractant signal, resulting in lower recognition and alveolar macrophage responses (12). The deposition of $20 \mathrm{~nm}$ particles is 2.7 times greater than $100 \mathrm{~nm}$ particles and 4.3 times greater than $200 \mathrm{~nm}$ particles (13). Higher deposition efficiencies occur in patients with asthma or chronic obstructive pulmonary disease than in healthy subjects, possibly due to decreased clearance ability (11). Kreyling et al. found that there was less than $25 \%$ clearance of 50 - and 100 $\mathrm{nm}$ particles during the first 24 hours after inhalation (12). The biopersistence of an inhaled particle is an important characteristic dictating the level of inflammation and tissue injury.

\section{Surface Area and Reactivity}

The surface area of inhaled nanoparticles favors the formation of free radicals (i.e. superoxide anions or hydroxyl radicals), which drive oxidative stress, an underlying mechanism that promotes inflammatory responses $(14,15)$. The ratio of surface atoms to total atoms or molecules increases exponentially with decreasing particle size, contributing to the surface reactivity (10). Oberdörster confirmed this by examining a difference in toxicities from nano-sized versus micron-sized titanium dioxide particles of the crystalline type, anatase, when instilled at the same mass dose. The nano-sized particles were more reactive than the micron-sized particles. The data was linearly correlated when the same experiments were performed at the same surface area dose (1).

The lung inflammatory response induced by particles is also dictated by shape, crystallinity, charge, surface modifications, and weathering (10). Many nanoparticles, such as combustion derived nanoparticles, agglomerate readily and move as an aggregate, which decreases particle number, but leaves surface area dosage unaffected. As particles undergo chemical interactions with components of the ambient air pollution cloud, there can be aging of the particles which may change their chemistries (14).

Other constituents of particle clouds also play a large role in pulmonary inflammation and fibrosis. Metals only need to be present in trace quantities to cause inflammatory effects, through the generation of reactive oxygen and nitrogen species (16). Additionally, the capacity of diesel exhaust particles to cause oxidative reactions in vivo has been attributed to their content of metals (17), polyaromatic hydrocarbons (18) and quinones (19). The component responsible for oxidative stress and subsequent pro-inflammatory signaling in diesel exhaust particles is principally the organic fraction (14). The organic fraction contains or can metabolize to components such as quinones, which readily produce reactive oxygen species (19). The surface area of the particle is important in the retention of these constituents, as they remain on or among the airborne particles.

\section{NANOPARTICLE-INDUCED PULMONARY FIBROSIS}

A complex set of tissue reactions must occur for the formation and accumulation of fibrous connective tissue that defines pulmonary fibrosis. Traditionally, fibrosis has been viewed as an irreversible process which varies from a restrictive ventilatory defect causing hypoxemia, pulmonary hypertension, and cor pulmonale, to the distortion of lung anatomy inducing bronchiectasis and chronic respiratory infection (20, 21). The pathogenesis of pulmonary fibrosis begins as an inflammatory response to injury when immune cells are excessively or inappropriately activated. These immune cells include macrophages and neutrophils that release toxic mediators, compromising epithelial integrity and promoting tissue injury. The normal repair process involves the recruitment and activation of mesenchymal cells resulting in extracellular matrix deposition, re-epithelialization and restoration of normal lung architecture. In certain patients, however, aberrant tissue remodeling and excessive matrix deposition leads to progressive scarring and fibrosis (22). In the context of particle inhalation it is likely that the inability to clear toxic particles from the lungs via mucociliary clearance or phagocytosis, as well as sustained exposure, may drive an exaggerated inflammatory response that leads to irregular tissue remodeling and fibrosis. Initiation of this cascade may occur due to interactions with alveolar macrophages, epithelial cells, or direct interactions with interstitial fibroblasts.

Nanoparticle exposure activates a number of cytokine/growth factor cascades via an increase in reactive oxygen species. The activation of receptor tyrosine kinases, mitogen-activated protein (MAP) kinases, and transcription factors, such as nuclear factor (NF)- $\mathrm{kB}$ and STAT-1, drive transcriptional activation and the expression of genes involved in inflammation and fibrosis $(15,23)$. Interleukin (IL)- $1 \beta$ and tumor necrosis factor (TNF)- $\alpha$ stimulation increase the expression of pro-fibrotic growth factors and their receptors. TNF- $\alpha$ causes an increase in the production 
of transforming growth factor (TGF)- $\beta 1$, a major stimulator of collagen deposition in fibroblasts (24). IL$1 \beta$ increases the expression of platelet-derived growth factor (PDGF)-AA and its receptor, PDGF receptor- $\alpha$, on lung fibroblasts (25). The coordinated secretion of PDGF-AA and PDGF receptor- $\alpha$ attracts fibroblasts from the interstitium and induces proliferation of myofibroblasts. The myofibroblasts form and organize immature collagenous tissue within the lung (15).

The organization of the immature fibrinous tissue with neovascularization, proliferation of myofibroblasts, deposition of increasing amounts of extracellular matrix components and the development of scarring contribute to a loss of tissue function corresponding to the extent of the fibrotic response (26). The removal of the airborne nanoparticle exposure causing lung injury could allow re-epithelialization, dependent upon the state of the basement membrane, collagen and elastic structure of the alveoli. The elimination of immature intra-lumenal collagenous tissue by the fibrinolytic system and apoptosis of the myofibroblasts are important for the re-epithelialization. However, the extent of lung injury may not preserve lung function (27).

The radiographic features of pulmonary fibrosis show patchy ground-glass opacities and numerous centrilobular nodules measuring 2 to $4 \mathrm{~mm}$ in diameter. Honeycombing may be seen in advanced fibrotic stages. A progressive decline in diffusion capacity and worsening gas exchange abnormalities with exercise are sensitive indicators of worsening pulmonary fibrosis. Pulmonary function tests show restrictive ventilatory impairment with a reduction in lung volumes, increased elastic recoil and decreased diffusion capacity (28).

\section{CLINICAL CASES OF PARTICLE-INDUCED PULMONARY FIBROSIS}

Most cases of particle-induced pulmonary fibrosis are classified as pneumoconioses. Pneumoconiosis, by definition, is an occupational lung disease caused by the inhalation of mineral and metallic particles and nanoparticle dusts. Pneumoconiosis is broken into two forms, fibrotic and nonfibrotic. Fibrotic pneumoconiosis includes silicosis (silica particles), coal worker's pneumoconiosis (washed coal particles), asbestosis (asbestos fibers), berylliosis (beryllium particles), and talcosis (magnesium silicate processing). Nonfibrotic pneumoconioses include siderosis (iron oxide particles), stannosis (tin oxide particles) and baritosis (barium sulfate particles). The most common forms of pneumoconioses are fibrotic, including silicosis, coal worker's pneumoconiosis, and asbestosis (29). In the US, death rates among males in 1968-1981 compared with 1982-2000 have shown a $36 \%$ decline in coal worker's pneumoconiosis, $70 \%$ decline for silicosis, but a $400 \%$ increase in asbestosis (5).

These clinical conditions are not solely influenced by the particle type after which they are named, but by multiple types of particles, varying in size and concentrations. In vivo and in vitro studies suggest that nano-sized particles are the most toxic particle component of particulate clouds, contributing most heavily to fibrogenicity $(8,30)$. Particle fibroses reviewed here are categorized according to the source of particles, which includes mineral dusts, combustionderived particles, and engineered nanoparticles. It is important to note that mineral and combustion-derived particle fibroses are occupational diseases mostly prevalent in developing nations. While there have been no documented cases of engineered nanoparticleinduced pulmonary fibrosis in humans, possibly due to exposure levels, exposure to engineered nanoparticles, such as carbon nanotubes, continues to increase as they become more integrated into technology $(31,32)$. The rest of this review is focused on clinical conditions of particle-induced pulmonary fibrosis, which supports nanoparticles as the most significant particle portion.

\section{MINERAL DUSTS}

Mineral dust composition varies by location, and can contain particles of crystalline silica, asbestos, carbon black and other molecules (33). These particles are the most common causes of particle-induced pulmonary fibrosis. For this reason, silicosis and asbestosis are examined in further detail.

\section{Silicosis}

Silicosis is caused by the inhalation of crystalline silica particles for extended periods of time. Silica is present in many different crystalline forms that vary in levels of fibrogenicity according to the degree of crystallization. Fibrogenicity increases from the less organized crystal structure of amorphous silica to the more organized crystal structures of quartz, cristobalite, and tridymite (33). As crystallinity plays a role in fibrogenicity, the large surface area per mass of silica nanoparticles allows for a greater production of reactive oxygen species catalyzed by the crystalline surface. The inhalation of silica can also lead to the development of bronchogenic carcinoma (34).

The pathology caused by silica particles involves their phagocytosis by alveolar macrophages. Silica uptake seems to be initiated by MARCO (macrophage receptor with collagenenous structure) as seen in mice by Hamilton et al. (35). Alveolar macrophages are damaged or activated and release cytotoxic oxidant or proteases and inflammatory cytokines such as TNF- $\alpha$, IL-1 and arachidonic acid metabolites, which provoke 
recruitment of inflammatory cells into the alveolar wall and alveolar epithelial surface $(22,36)$. The generation of oxidants by silica nanoparticles and silica-activated immune cells results in additional macrophage apoptosis, lung damage, inflammation, and cell transformation. The pro-inflammatory cytokines released during inflammation by macrophages and potentially by neutrophils, mast cells and Blymphocytes participate in the exaggerated deposition of matrix protein and the persistence of transformed cells characterizing silicosis (22). Direct interaction of fibroblasts with silica has also been shown to drive cyclooxygenase-2 and prostaglandin E2 expression, creating a pro-fibrotic cytokine milieu (37). Silicotic nodules caused by silica inhalation consist of fibrotic lesions and are distributed in the upper part of the lungs. The nodule is termed an "onion skin lesion" with collagen fibers concentrically arranged, and dust-laden macrophages surrounding the mature collagen $(34,29)$. Thin-film computed tomography show numerous bilateral centrilobular nodular ground-glass opacities, multifocal patchy ground-glass opacities and consolidation (29, 38). Figure 1 shows a chest radiograph and $\mathrm{CT}$ scan from a male with silicosis who worked in stonecutting for twenty-five years.

\section{Asbestosis}

Asbestosis is induced by the inhalation of asbestos fibers. Asbestos is a fibrous silicate mineral used in industry for its heat resistance and tensile strength. The inhalation of asbestos can also lead to the development of other pulmonary diseases, such as benign pleural effusion and plaques, mesothelioma and bronchogenic carcinoma (39).

Deposition of asbestos is based upon its aerodynamic diameter, and the ability of the fiber to align with the airway. The biopersistence and accumulation of asbestos is important in dictating the level of fibrotic response (16). The inhalation of asbestos fibers damages alveolar macrophages and epithelial cells, causing them to release inflammatory mediators and growth factors (38). NF- $\mathrm{kB}-$, protein kinase C- and MAPK-dependent inflammatory pathways are activated as a result. The MAPK ERK1/2 is selectively phosphorylated in lung epithelium after the inhalation of asbestos. The duration of ERK1/2 activation dictates the toxic response to asbestos and related production of reactive oxygen and nitrogen species. ERK1/2 is linked to TNF $\alpha$ and TGF- $\beta 1$ expression, resulting in inflammation and fibrogenesis (39). Asbestosis appears to be histologically and radiographically similar to idiopathic pulmonary fibrosis with honeycombing being a common feature of late stage asbestosis (29).

\section{COMBUSTION-DERIVED PARTICLES}

Combustion-derived nanoparticles cover a large variety of nanoparticles, including diesel exhaust particles, welding-fume particles, fuel oil ash, and coal fly ash (14). These nanoparticles are largely nonfibrogenic, except occupational exposure to coal fly ash, oil fly ash, and welding fume nanoparticles in the cases of boilermakers (40). Diesel exhaust particles have been found to cause fibrotic events in rats as a result of lung overload, but humans are unlikely to ever experience high levels comparable to those issued to experimental rats $(14,41)$. Combustion-derived nanoparticles may be soluble and release transition metals or organics as their primary pro-inflammatory mechanism. Both transition metals and organics can undergo complex cyclical chemical reactions in the
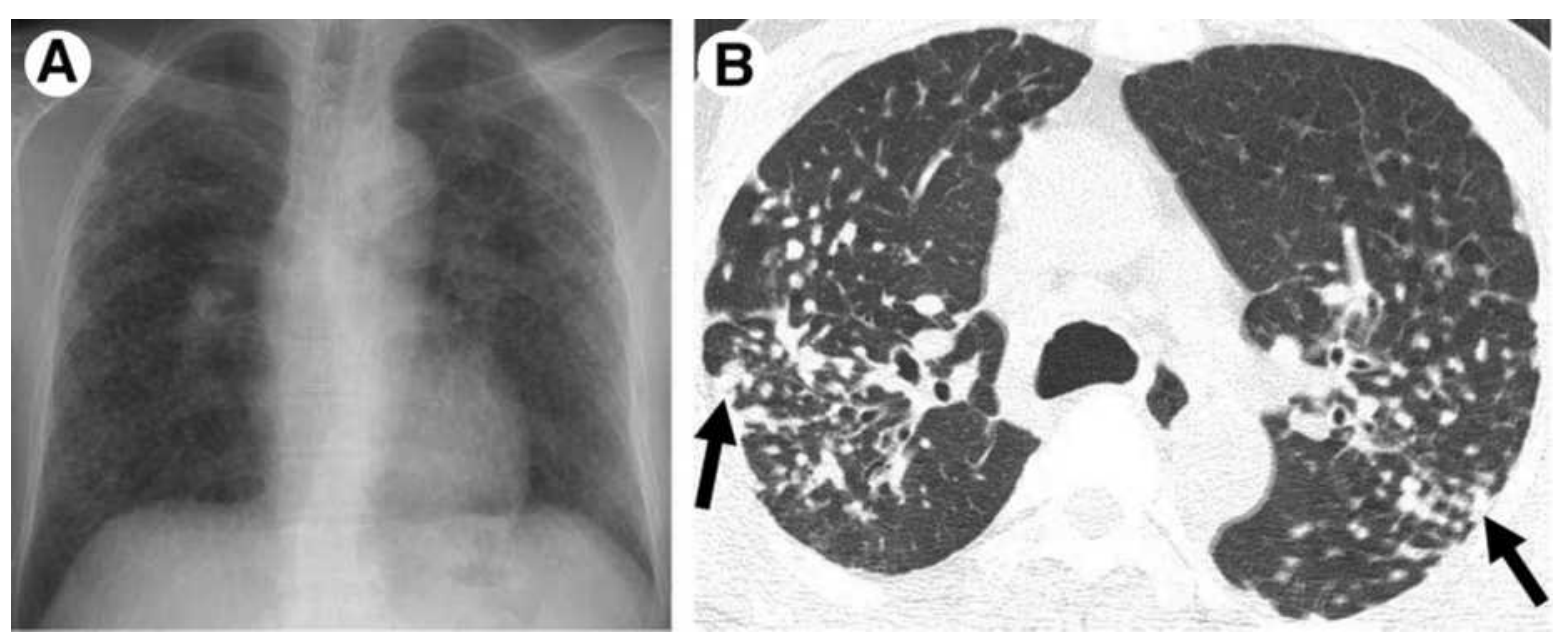

Figure 1: Silicosis in a 56-year-old male who worked in stonecutting for 25 years. (A) Chest radiograph shows multiple variable-sized nodular lesions in both lungs, predominantly in the upper and middle zones. (B) Axial thin-section CT scan (1.0-mm-thick section) obtained at the level of the azygos arch shows multiple small nodules with a perilymphatic (centrilobular plus subpleural) distribution in the upper lobe of both lungs. Note the tendency toward coalescence of the nodules in the lung periphery (arrows). With copyright permission from RSNA and Dr. Kyung Soo Lee (29). 
milieu of the lungs that lead to the production of free radicals such as superoxide anion or hydroxyl radical (19). Fibrosis induced by welding fumes and oil fly ash (OFA) are examined in this section.

\section{Welding-Induced Pneumoconiosis}

Welding involves the use of electrical currents in excess of 200 amperes and a space-filling metal rod to join two metal objects together. In the process, some of the liquefied metal is aerosolized (42). Most welding materials are alloy mixtures of metals characterized by different steels that may contain iron, manganese, silica, chromium and nickel (43).

Exposure to welding fume nanoparticulate matter in humans is associated with inflammatory cytokine increases in the bronchoalveolar lavage fluid (44). Rats exposed to welding fumes have shown marked pulmonary inflammatory responses and lipid peroxidation indicative of oxidative stress (45). In addition, epithelial cells exposed to welding fumes or the transition metals associated with them exhibited oxidative stress which caused MAPK-dependent NF- $\kappa \mathrm{B}$ and AP-1 activation leading to IL-8 upregulation (46). Thus, the soluble transition metals appear to be the primary mechanism of oxidative stress and inflammation (14).
Hull and Abraham examined cases of aluminumwelding particle-induced pneumoconiosis, and found areas of severe dense fibrosis which were interspersed with macrophages containing particles (42). The lung parenchyma display focally dense fibrosis, more severe in the upper lobes, with peripheral honeycombing sparing the lung bases. Figure 2 shows dense fibrotic tissue with alveolar macrophages encapsulating welding particles from an aluminum welder (42).

\section{Oil Fly Ash}

Human exposure to oil fly ash (OFA), the inorganic residue from burning carbonaceous materials, occurs where workers, specifically boilermakers, are engaged in the maintenance of oil-fired boilers. Boilermakers that are exposed to OFA have shown dose-dependent decreases in pulmonary function caused by pulmonary inflammation (47).

In these cases of exposure to OFA, the most toxic component appears to be the vanadium pentoxide on the surface of the particle or among the particles. Vanadium pentoxide, a transition metal derived from the burning of petrochemicals, caused the rapid onset of fibrosis in rats (48). In vivo studies by Bonner et al. have shown fibrotic responses through the intratracheal instillation of vanadium pentoxide (48-49). Vanadium compounds
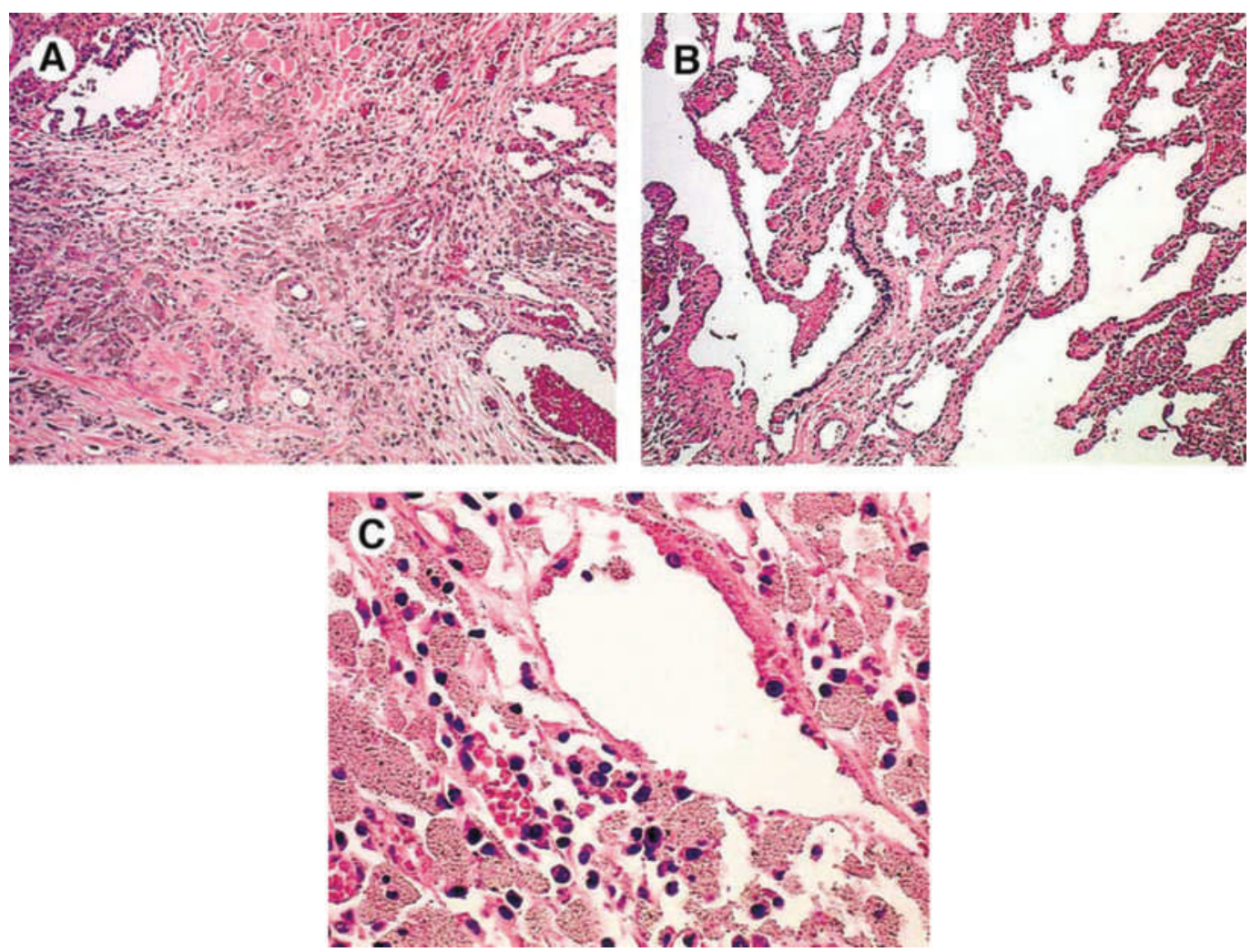

Figure 2: Case 1, LM: (A) Dense fibrotic area of the lung with many aluminum-containing macrophages. (Original magnification $x 10$.) (B) Lung parenchyma showing peribronchiolar and interstitial aluminum-containing macrophages. (Original magnification $\mathrm{x}$ 10.). (C) Aluminumcontaining macrophages in lymph node. (Original magnification x 180.) (42). With copyright permission from Elsevier and Dr. Jerrold Abraham. 
activate many intracellular signaling pathways via the generation of hydrogen peroxide and many other reactive oxygen species (50). The fibrogenicity of the vanadium pentoxide relates to particle size, as the extent of the surface area is indicative of the chemical carrying nature of the particle.

\section{ENGINEERED NANOPARTICLES}

Engineered nanoparticles are particles manufactured to increase surface area and conductivity for materials or to act as delivery agents. These nanoparticles are made from a variety of materials including oxides, metals, carbon, polymers and lipids. Many polymer and lipid nanoparticles have non-reactive surfaces; however, many oxide, metal, and carbon particles are quite reactive (51). The most relevant fibrogenic nanoparticles are carbon nanotubes, which have been compared to asbestos in terms of toxicity. There have been no documented human cases of fibrosis from carbon nanotubes, but exposure to carbon nanotubes is on a steady rise because of their huge potential in industrial applications (6). As the manufacturing costs decrease, it has been predicted that hundreds or thousands of tons of carbon nanotubes could be produced in five to ten years (52).

\section{Carbon Nanotube-induced Fibrosis}

Carbon nanotubes have very unique electrical, mechanical and thermal properties, which allow them to have potential application in the electronics, computer and aerospace industries. Carbon nanotubes are found to deposit in the alveolar ducts by aligning their long dimension to the direction of the airstream. Nanotubes longer than $20 \mu \mathrm{m}$ can cause the same types of pathology as asbestos and other fibers. Carbon nanotubes have a larger surface area than nanoparticles, thus having a higher proclivity to cause more effects. In addition, they have a tendency to get contaminated with metals (53).

Muller et al. were able to show the occurrence of fibrosis in rats after the instillation of multi-wall carbon nanotubes, ground and intact. They ground up carbon nanotubes, and induced a higher degree of inflammation than with intact multi-wall carbon nanotubes. The assessment of lung hydroxyproline (OH-proline) and soluble type I collagen showed a dose-dependent increase in extracellular matrix expression. Histopathologically, collagen-rich granulomas formed in the bronchi, which partially or completely blocked the bronchial lumen. The granulomas reepithelialized, organized around the nanotubes and formed multinuclear giant cells as well as macrophages and other mononuclear inflammatory cells (6). Further carbon nanotube studies showed that carbon nanotubes caused significant increases in lung PDGF mRNA (30). All documented in vivo studies of carbon nanotubes have been by instillation, rather than inhalation. Thus, the fibrogenic effects of the nanotubes could be a result of the complexes formed during the instillation and may not represent a respirable cloud (53).

Lam et al. showed that carbon nanotubes produce profibrogenic lesions similar to those of toxic silica particles (32). Human pulmonary fibrosis caused by inhalation of carbon nanotubes may be very similar to silicosis cases. Overall, in vivo studies point to the induction of fibrosis in humans. Limiting exposure is essential in containing the occurrence of pulmonary fibrosis.

\section{SUMMARY AND FUTURE OUTLOOK}

We review particle-induced pulmonary fibrosis and support that nanoparticles are a significant, if not the most significant, particle component contributing to pulmonary fibrosis. The two main factors that contribute most heavily to particle-induced lung diseases are particle surface area and the reactivity or intrinsic toxicity of that surface. In addition, the size of nanoparticles allows them to get deposited in lungs at a greater efficiency than particles of larger sizes, yielding a higher biopersistency.

The ability to produce free radicals is important in the induction of inflammation and fibrosis. The inhalation of nanoparticles can induce fibrosis, based upon the time of exposure, exposure concentration, and ability to produce free radicals. By providing insight into clinical and experimental cases of particle-induced pulmonary fibrosis, we hope to have shown the danger of nanoparticle inhalation.

\section{ACKNOWLEDGMENT}

This research was supported by the UCD College of Life Sciences and College of Engineering, Mathematical and Physical Sciences to facilitate USIreland collaboration.

\section{REFERENCES}

(1) Oberdörster G. Toxicology of Ultrafine Particles: In Vivo Studies. Philosophical Transactions of the Royal Society in London Part A 200; 358: 2719-2740.

(2) Lkhasuren O, Takahashi K, Dash-Onolt, L. Occupational Lung Diseases and the Mining Industry in Mongolia. International Journal of Environmental Occupational Health 2007; 13: 195 201

(3) Wang XR and Christiani DC. Occupational Lung Disease in China. International Journal of Occupational and Environmental Health 2003; 9: 320-325.

(4) Public Health Agency of Canada. Life and Breath: Respiratory Disease in Canada 2007; 32-33.

(5) Attfield MD, Wood JM, Antao VC, Pinheiro GA. Changing 
Patterns of Pneumoconiosis Mortality - United States, 19682000. Morbidity and Mortality Weekly Report 2004; 292: 795796.

(6) Muller J, Huaux F, Moreau N. Respiratory Toxicity of MultiWall Carbon Nanotubes. Toxicology and Applied Pharmacology 2005; 207: 221-231.

(7) Donaldson K, Stone V, Borm PJ, et al. Oxidative stress and calcium signaling in the adverse effects of environmental particles (PM10). Free Radical Biology and Medicine 2003; 34:1369-1382.

(8) Duffin R, Mills NL, Donaldson, K. Nanoparticles - A Thoracic Toxicology Perspective. Yonsei Medical Journal 2007; 48: 561572.

(9) Gwinn MR, Vallyathan V. Nanoparticles: Health Effects - Pros and Cons. Environmental Health Perspectives 2006; 114: 18181825.

(10) Oberdörster G, Oberdörster E, Oberdörster J. Nanotoxicology: An Emerging Discipline Evolving from Studies of Ultrafine Particles. Environmental Health Perspectives 2005; 113: 823 839.

(11) Anderson PJ, Wilson JD, Hiller FC. Respiratory Tract Deposition of Ultrafine Particles in Subjects with Obstructive or Restrictive Lung Disease. Chest 1990; 97: 1115-1120.

(12) Kreyling WG, Semmler-Behnke M, Möller W. Ultrafine Particle-Lung Interactions: Does Size Matter? Journal of Aersosol Medicine 2006; 19: 74-83.

(13) Stahlhofen W, Rudolf G, James AC. Intercomparison of experimental regional aerosol deposition data. Journal of Aerosol Medicine 1989 ; 2: 285-308.

(14) Donaldson K, Tran L, Jimenez LA, et al. Combustion-derived nanoparticles: A review of their Toxicology following Inhalation Exposure. Particle and Fibre Toxicology 2005; 2: 10.

(15) Bonner JC. Lung Fibrotic Responses to Particle Exposure. Toxicologic Pathology 2007; 35: 148-153.

(16) Donaldson K, Tran CL. Inflammation caused by Particles and Fibers. Inhalation Toxicology 2002; 14: 5-27.

(17) Mudway IS, Stenfors N, Duggan ST, et al. An in vitro and in vivo Investigation of the Effects of Diesel Exhaust on Human Airway Lining Fluid Antioxidants. Archives of Biochemistry and Biophysics 2004; 423:200-212.

(18) Baulig A, Garlatti M, Bonvallot V. Involvement of Reactive Oxygen Species in the Metabolic Pathways triggered by Diesel Exhaust Particles in Human Airway Epithelial Cells. American Journal of Physiology Lung Cellular and Molecular Physiology 2003; 285: L671-L679.

(19) Squadrito GL, Cueto R, Dellinger B, Pryor WA. Quinoid Redox Cycling as a Mechanism for Sustained Free Radical Generation by Inhaled Airborne Particulate Matter. Free Radical Biology and Medicine 2001; 31: 1132-1138.

(20) Zisman DA, Keane MP, Belperio JA, Streiter RM, Lynch JP III. Pulmonary Fibrosis. Methods in Molecular Medicine 2005; 117: 3-44.

(21) Dheda K, Booth H, Huggett JF, Johnson MA, Zumla A, Rook JA. Lung remodeling in pulmonary tuberculosis. Journal of Infectious Diseases 2005; 192: 1201-1209.

(22) Huaux F. New Developments in the Understanding of Immunology in Silicosis. Current Opinion in Allergy and Clinical Immunology 2007; 7: 168-173.

(23) Bonner JC. The Epidermal Growth Factor Receptor at the Crossroads of Airway Remodeling. American Journal of Physiology 2002; 283: L528-L530.

(24) Sime PJ, Marr RA, Gauldie D et al. Transfer of tumor necrosis factor- $\alpha$ to Rat Lung induces Severe Pulmonary Inflammation and patchy Interstitial Fibrogenesis with Induction of Transforming Growth Factor $-\beta 1$ and myofibroblasts. American Journal of Pathology 1998; 153: 825-832.
(25) Lindroos PM, Coin PG, Badgett A, Morgan DL, Bonner JC. Alveolar Macrophages stimulated with Titanium Dioxide, chrysotile asbestos and Residual Oil Fly Ash up-regulate the PDGF Receptor- $\alpha$ on Lung Fibroblasts through an IL-1 $\beta$ dependent Mechanism. American Journal of Respiratory Cellular and Molecular Biology 1997; 16: 283-292.

(26) Kumar V, Abbas AK, Fausto N. Tissue renewal and repair: regeneration, healing and fibrosis. In: Pathological Basis of Disease, 7th edn, pp. 87-118. 2005. Philadelphia, PA: Elsevier Saunders.

(27) Wallace WAH, Fitch PM, Simpson AJ, Howie SEM. Inflammation-associated remodeling and fibrosis in the lung - a process and an end point. Int. J. Exp. Path. 2007; 88: 103-110.

(28) Mohr LC. Hypersensitivity Pneumonitis. Currect Opinion in Pulmonary Medicine 2004; 10: 401-411.

(29) Chong S, Soo Lee K, Jin Chung M, Han J, Jung Kwon O, Sung Kim T. Pneumoconiosis: A Comparison of Imaging and Pathologic Findings. RadioGraphics 2006; 26: 59-77.

(30) Donaldson K, Tran CL, MacNee W. Deposition and effects of fine and ultrafine particles in the respiratory tract. The European Respiratory Monograph 2002; 7: 77-92.

(31) Mangum JB, Turpin EA, Antao-Menezes A, Cesta MF, Bermudez E, Bonner JC. Single-Walled Carbon Nanotube (SWCNT)-induced interstitial fibrosis in the lungs of rats is associated with increased Levels of PDGF mRNA and the formation of unique Intercellular Carbon Structures that bridge Alveolar Macrophages In Situ. Particle and Fibre Toxicology 2006; 3: 15 .

(32) Lam CW, James JT, McCluskey R, Hunter RL. Pulmonary Toxicity of Single-Wall Carbon Nanotubes in Mice 7 and 90 Days after Intratracheal Instillation. Toxicological Sciences 2004; 77: 126-134.

(33) Heppleston AG. Minerals, Fibrosis, and the Lung. Environmental Health Perspectives 1991; 94: 149-168.

(34) Fujimura N. Pathology and pathophysiology of pneumoconiosis. Current Opinion in Pulmonary Medicine 2000; 6: $140-144$

(35) Hamilton RF, Thakur SA, Mayfair JK, Holian A. MARCO Mediates Silica Uptake and Toxicity in Alveolar Macrophages from C57BL/6 Mice. J Bio Chem 2006; 281: 34218-34226.

(36) Mossman BT, Churg A. Mechanisms in the Pathogenesis of Asbestosis and Silicosis. American Journal of Respiratory Critical Care Medicine 1998; 157: 1666-1680.

(37) O'Reilly KMA, Phipps RP, Thatcher TH, Graf BA, Van Kirk J, Sime PJ. Crystalline and Amorphous Silica differentially regulate the Cyclooxygenase-prostaglandin pathway in Pulmonary Fibroblasts: Implications for Pulmonary Fibrosis. American Journal of Physiology Lung and Cellular Molecular Physiology. 2005; 288: L1010-L1016.

(38) Marchiori E, Ferreira A, Müller NL. Silicoproteinosis: highresolution $\mathrm{CT}$ and histologic findings. Journal of Thoracic Imaging 2001; 16: 127-129.

(39) Mossman BT, Borm PJ, Castranova V, Costa DL, Donaldson K, Kleeberger SR. Mechanisms of action of inhaled fibers, particles and nanoparticles in lung and cardiovascular diseases. Particle and Fiber Toxicology 2007; 4: 1-10.

(40) Woodin MA, Liu Y, Neuberg D, Hauser R, Smith TJ, Christiani DC. Acute Respiratory Symptoms in Workers Exposed to Vanadium-Rich Fuel-Oil Ash. American Journal of Industrial Medicine 2000; 37: 353-363.

(41) Tran CL, Buchanan D, Cullen RT, et al. Inhalation of Poorly Soluble Particles: II. Influences of Particle Surface Area on Clearance and Inflammation. Inhalation Toxicology 2000; 12: 101-115.

(42) Hull MJ, Abraham JL. Aluminum Welding Fume-Induced Pneumoconiosis. Human Pathology 2002; 33: 819-825. 
(43) Antonini JM. Health Effects of Welding. Critical Reviews in Toxicology 2003; 33: 61-103.

(44) Blanc PD, Boushey HA, Wong H, Wintermeyer SF, Bernstein MS. Cytokines in Metal Fume Fever. American Review of Respiratory Disease 1993; 147: 134-138.

(45) Taylor MD, Roberts JR, Leonard SS, Shi X, Antonini JM. Effects of Welding Fumes of Differing Composition and Solubility on free Radical Production and Acute Lung Injury and Inflammation in Rats. Toxicological Sciences 2003; 75: 181-191.

(46) McNeilly JD, Heal MR, Beverland IJ, et al. Toxicological Applied Pharmacology 2004; 196: 95-107.

(47) Ghio AJ, Silbajoris R, Carson JL, Samet JM. Biologic Effects of Oil Fly Ash. Environmental Health Perspectives 2002; 110: 8994.

(48) Bonner JC, Rice AB, Moomaw CR, Morgan DL. Airway Fibrosis in Rats induced by Vanadium Pentoxide. American Journal Lung Cellular and Molecular Physiology 2000; 278: L209-L216.

(49) Ress NB, Chou BJ, Renne RA, et al. Carcinogenicity of Inhaled
Vanadium Pentoxide in F344/N Rats adn B6C3F1 Mice. Toxicological Sciences 2003; 74: 287-296.

(50) Ingram JL, Rice AB, Santos J, Van Houten B, Bonner JC. Vanadium-induced HB-EGF expression in Human Lung Fibroblasts is Oxidant Dependent and requires MAP Kinases 2003; 284: 774-782.

(51) Bermudez E, Mangum JB, Wong BA, Asgharian B, Hext PM. Pulmonary Responses of Mice, Rats, and Hamsters to Subchronic Inhalation of Ultrafine Titanium Dioxide Particles. Toxicological Sciences 2004; 77: 347-357.

(52) Taubes G. Nanotechnology. An interview with Dr. Richard Smalley. ISI Essential Science Indicators Special Topics. 2002.

(53) Donaldson K, Aitken R, Tran L et al. Carbon Nanotubes: A Review of their Properties in Relation to Pulmonary Toxicology and Workplace Safety. Toxicological Sciences 2006; 92: 5-22.

James Byrne is an undergraduate student from the University of Texas in Austin. He will receive a B.Sc. in Biomedical Engineering in May 2008. His current research focus is on cancer therapeutics and nanoparticle drug delivery systems.

John Baugh is a lecturer in Medicine at University College Dublin, Ireland. He received a B.Sc. (Hons) in Pharmacology and a PhD from the University of Bath, UK. His current research focuses on the mechanisms of aberrrant tissue remodeling in pulmonary fibrosis. 